Voix et Images

voixetimages

\title{
Les Oeuvres complètes de Lahontan
}

\section{Gilles Thérien}

Volume 17, numéro 3 (51), printemps 1992

Paul-Marie Lapointe

URI : https://id.erudit.org/iderudit/200984ar

DOI : https://doi.org/10.7202/200984ar

Aller au sommaire du numéro

\section{Éditeur(s)}

Université du Québec à Montréal

\section{ISSN}

0318-9201 (imprimé)

1705-933X (numérique)

Découvrir la revue

\section{Citer cet article}

Thérien, G. (1992). Les Oeuvres complètes de Lahontan. Voix et Images, 17(3), 520-529. https://doi.org/10.7202/200984ar d'utilisation que vous pouvez consulter en ligne.

https://apropos.erudit.org/fr/usagers/politique-dutilisation/ 
Recherche

\section{Les CEuvres complètes de Lahontan}

Gilles Thérien, Université du Québec à Montréal

La publication de l'édition critique des CEuvres complètes de Lahontan ${ }^{1}$ par Réal Ouellet et Alain Beaulieu, dans la collection de la 
Bibliothèque du Nouveau Monde, met un terme à une entreprise de presque vingt ans. Le mode de travail, choisi par l'éditeur principal qui a réuni un "collectif interdisciplinaire", montre que ce genre d'édition, où les manuscrits manquent, où les traditions de lecture sont déficientes, où la littérature critique est inconsistante faute de pouvoir identifier correctement ses sources, où l'histoire même n'est pas d'une aide absolue lorsque, près de trois siècles plus tard, on tente de rassembler les pièces du puzzle autour d'un personnage dont l'importance est loin d'être évidente, que ce genre d'édition, dis-je, se conçoit sous le mode de la collaboration, du partage des tâches et d'une nette tendance à l'exhaustivité. Voilà! Le travail est achevé, je l'ai devant moi et je dois en faire un compte rendu d'ici vingt ans... puisque le texte fait bien en tout 1474 pages et 3798 notes dont, heureusement, un bon nombre ne constituent que des renvois à d'autres notes ou à différents extraits des textes.

L'avantage le plus évident de cette édition est, bien sûr, de nous fournir l'œuvre complète de Lahontan, c'est-à-dire non seulement les Nouveaux Voyages, les Mémoires, la Suite du voyage de l'Amérique qui comprend les fameux Dialogues, mais encore divers textes et lettres dont l'intérêt contextuel est important. Une chronologie annotée, accompagnée d'une documentation en appendice, permet de jeter toute la lumière disponible et nécessaire sur-ce-très -curieux personnage, y compris sur la date de son décès et, aussi, assez curieusement, sur son état d'esprit religieux à ce moment. L'ignorance de ces éléments a trop souvent permis des conclusions hâtives et abusives sur une œuvre jugée aussi fantaisiste que son auteur. Dans l'introduction de 1973 à la publication des Dialogues aux Éditions sociales, par ailleurs excellente, Maurice Roelens s'interrogeait encore sur la date de la mort de Lahontan qu'il fixait aux environs de 1715. Maintenant, 276 ans après le fait, nous sommes fixés: * Le 21 avril 1716 est mort le très illustre seigneur baron de Lahontemps, de nationalité française, avant 'd'avoir pu faire ses pâques comme il l'avait souhaité; l'inhumation a eu lieu le 22 du même mois, vers le soir. Qu'il repose en paix!* (p. 1153) L'extrait est celui du registre de l'église Saint-Clément. Cette précieuse information ne vient toutefois pas combler toutes les lacunes de notre savoir sur l'existence mouvementée de Lahontan depuis son retour en Europe en 1694. Autant nous semblons être bien informés de son séjour en Amérique, autant la seconde partie de sa vie n'est-elle connue que par des bribes d'information même au moment où il acquiert une popularité importante avec la publication, à partir de 1702 , de ses relations de voyages et, plus particulièrement, de ses dialogues avec le très sage Adario. C'est dire comment le travail 
de l'édition, dans toute sa minutie et sa volonté d'explorer tout ce qui touche au phénomène Lahontan nous permet de prendre la meilleure mesure possible du contexte dans lequel a été élaboré l'œuvre du malheureux baron.

Suivant en cela l'excellent protocole établi par la Bibliothèque du Nouveau Monde, les informations les plus synthétiques nous sont fournies dans l'introduction à quatre voix, où celles de Réal Ouellet et Alain Beaulieu dominent. Longue de 189 pages, accompagnée de 781 notes, l'introduction suit le modèle classique: présentation de l'auteur, contexte historique, présentation de l'œuvre et examen de son importance tant sur le plan documentaire que sur le plan philosophique; sa valeur littéraire et, évidemment, sa fortune de même que les polémiques qui l'ont entourée. Des indications sur les manuscrits de même que sur le contexte des diverses éditions complètent les informations plus textuelles. Une section est enfin consacrée à la faune et à la flore telles que représentées dans l'œuvre.

L'introduction a visiblement pour but de réhabiliter, pour les lecteurs que nous sommes, Lahontan et son œuvre et ce, sur trois plans: au plan de l'historiographie de la Nouvelle-France, au plan de l'histoire des idées et au plan de l'institution littéraire. Plusieurs arguments foumis par les éditeurs pourraient être définis sous l'étiquette * défense et illustration ", trame de fond qui anime toute l'introduction. Ainsi, le personnage de Lahontan est présenté comme un jeune homme sympathique, quelque peu éprouvé par un destin familial malheureux qui l'a laissé sans le sou et l'oblige à venir faire fortune en Amérique. Déjà!... L'introduction, combinée à la chronologie et aux divers documents disponibles, campe le personnage d'un jeune homme au seuil de la maturité qui vient séjoumer une dizaine d'années plus ou moins en NouvelleFrance. Il est né le 9 juin 1666 et débarque à Québec en novembre 1683 avec trois compagnies de marine, à titre peut-être de volontaire ou de cadet. Il n'obtiendra une reconnaissance militaire qu'en 1687, soit quatre ans plus tard. C'est cette année qu'il s'enfonce dans le territoire de la région des Grands Lacs. Il revient à Montréal le 10 juillet 1689 juste à temps pour le massacre de Lachine. En novembre 1690, il fait un saut en France pour ses affaires personnelles, qu'il n'arrivera pas à régler, mais il est nommé capitaine réformé et revient à Québec en septembre 1691. Il repart pour la France en juillet 1692, fait escale à Plaisance, où il se distingue dans des faits d'armes. En novembre, il poursuit son voyage en France où il réussira à se faire nommer Lieutenant du Roi. En mai 1693, il revient à Plaisance y occuper sa lieutenance. Il s'y brouille avec le gouverneur au nom prédestiné de Brouillan et rembarque pour la France en décembre. C'est la fin de son séjour en Nouvelle-France et 
c'est le début de son errance européenne qui se terminera seulement à sa mort. Si je me suis permis de refaire ce parcours biographique, c'est pour faire ressortir certains faits de l'édition qui méritent à mon sens une attention un peu plus particulière.

En fait, Lahontan passe moins de dix ans en Nouvelle-France. Son voyage dans l'Ouest ne dure que deux ans en incluant l'aller et le retour, les passages au fort Frontenac, au fort Saint-Joseph, son voyage à la rivière Longue qui dure presqu'un an et son séjour à Michillimakinac. Son séjour en Nouvelle-France ne dure donc qu'environ huit ans qu'il partage entre Québec, Montréal, l'expédition vers l'Ouest et le voyage à Terre-Neuve, séjour fructueux où il fait de l'exploration et rencontre des personnages importants, dont Frontenac, qui l'invite à sa table. C'est de ce séjour qu'il tire la matière de la plus grande partie de son œuvre. À ces considérations, ajoutons-en une autre qui n'est pas sans importance, l'âge. Il arrive à Québec à 17 ans et quitte la Nouvelle-France à 27 ans. Il a 21 ans quand il voyage dans l'Ouest... Réal Ouellet cite dans son introduction un extrait de la préface de la Suite du voyage: aJe passai de l'âge de 15 à 16 ans en Canada d'où j'eus le soin d'entretenir toujours un commerce de lettres avec un vieux Parent.... (p. 28) En fait, comme le montre la chronologie, il avait 17 ans révolus au moment de son arrivée en Nouvelle-France. Sympathique personnage qui, en 1703 , ne se souvient plus très bien de l'âge qu'il avait lorsqu'il est arrivé en NouvelleFrance vingt ans plus tôt. C'est ce genre de détail qui laisse perplexe. Il y en a d'autres, nous y reviendrons.

Suivons d'abord l'argumentation des éditeurs sur l'élaboration de l'œuvre. Celle-ci est articulée principalement sur cette correspondance entretenue avec un parent jamais identifié. Réal Ouellet indique bien comment il est peu probable que cette correspondance ait pu être tenue dès 1683. Elle donne tous les signes d'avoir été remaniée et conserve une sorte d'unité de ton assez invraisemblable sans compter les renvois, les précautions d'énonciation que Réal Ouellet souligne si justement. J'ajouterais un autre argument à l'appui du caractère fictif de cette correspondance: son irrégularité. Cette correspondance n'est pas suivie au sens habituel du terme. Elle tient plus du journal, qui sert à consigner, que de l'acte de communication. En outre, les détails plus personnels touchent toujours les questions familiales et juridiques de Lahontan, jamais de son correspondant. En somme, il y a certainement eu une correspondance, mais son intérêt principal était, vraisemblablement, de suivre le cours des affaires familiales. C'est d'ailleurs ces intérêts qui amènent Lahontan à se rendre en France d'où il écrit toujours à son mystérieux correspondant sans même le visiter. En ce sens, compte tenu des informations fournies par l'édition critique, j'imaginerais volontiers 
une correspondance d'affaires qui, par la suite, deviendra le prétexte à l'élaboration d'une correspondance plus importante, mais qui cache sous ce prétexte la volonté de-répéter le schéma des Relations des jésuites: une lettre à la fois privée, qui s'adresse au supérieur, et publique, puisqu'elle est immédiatement publiée. La répartition des lettres par périodé illustre la même idée. En 1683, il écrit une seule lettre, six en 1684, deux en 1685 , une en 1686 , trois en 1687 , deux en 1688 , trois en 1689 , une seule en 1690, trois en 1691, mais deux sont écrites de France. Trois autres lettres en 1692, 1693 et 1694, mais les trois écrites en Europe. En somme, sur les vingt-cinq lettres écrites par Lahontan, cinq sont européennes. Les six premières lettres sont courtes et suivent le modèle général de la relation de voyage: voyage lui-même, arrivée, état des lieux... La septième lettre, qui est un peu longue, contient la harangue faite par La Grangula à La Barre, harangue qui se retrouve dans les archives des colonies et dans les inédits de Lahontan. Cette harangue me semble importante et ne peut être absolument dissociée de la manière des dialogues qui seront ensuite attribués à Adario. Aussi, les cinq lettres européennes de Lahontan nous plongent-elles un peu plus avant dans l'ombre de l'auteur. Est-il véritablement l'émissaire de Frontenac ou simplement un entrepreneur qui cherche à devenir lobbyiste à la Cour? Difficile à dire! Il demeure que la fin de sa correspondance sur la Nouvelle-France marque le début de sa disgrâce et de son errance.

Les Mémoires, qui accompagnent la correspondance, sont en fait la reprise des mêmes données, sous une forme mieux organisée, pour qui veut avoir une vision globale de la situation en Nouvelle-France sans avoir à suivre tout le parcours chronologique de l'auteur. Ils contiennent aussi des informations que Lahontan a dû lire ou entendre, en particulier sur tout ce qui précède sa venue en NouvelleFrance. La complémentarité des Mémoires et des Nouveaux Voyages est telle qu'il est difficile de ne pas les voir tous les deux faire partie d'une même stratégie de l'auteur qui, en fait, par tous les moyens, cherche à reprendre du service auprès d'un gouvernement, d'abord le sien, mais ensuite tout autre gouvernement qui voudrait bien l'employer, l'espagnol, l'anglais, le danois, etc. Lorsqu'on les replace dans leur contexte, ces Mémoires, lus en dehors des Nouveaux Voyages, ont l'avantage de jeter un vaste regard sur la vie quotidienne des Indiens, un vaste regard laïque, le premier à nous avoir été transmis si l'on fait exception de celui de Nicolas Perrot écrit autour de 1700, mais qui ne sera publié qu'en 1864 . Les sujets que les jésuites n'osaient aborder, comme la sexualité, y trouvent place.

La pièce maîtresse de l'œuvre de Lahontan demeure ses Dialogues avec Adario. $\mathrm{Au} \mathrm{XVIII}{ }^{\mathrm{e}}$ siècle, ceụx qui avaient perdu leur l'intérêt 
pour les relations de voyage en Amérique du Nord se trouvent devant quelque chose de bien différent lorsqu'ils abordent les propos d'Adario, le sage Huron, dont le vrai nom serait Kondiaronk dit le Rat. Il faut noter ici que Lahontan ne nomme jamais Adario Kondiaronk, ce qui ne devrait pas manquer d'étonner puisqu'il prétend le connaître très bien. Et, $s^{\prime}$ il le connaissait sous son vrai patronyme, il apparaît encore plus curieux de le voir utiliser l'appellation française, "le Rat", qui n'a rien d'élogieux, ou encore "Adario", qui ressemble plus à un nom de théâtre. Les éditeurs ont raison d'insister sur le caractère novateur de ce dialogue et, à la suite de Maurice Roelens, d'affirmer que ces écrits

sont à bien des égards représentatifs de cette première vague de philosophie critique, souvent plus radicale que la philosophie des Lumières, qui a déterminé entre 1685 et 1715 ce qu'il est convenu d'appeler depuis Paul Hazard la - crise de la conscience européenne . (p. 54, $\mathrm{n}^{\circ}$ 144)

Le travail d'édition critique porte aussi sur les variantes imposées par la réécriture de Gueudeville ${ }^{2}$. Cela est vrai pour tous les textes, mais ce l'est encore plus dans le cas des Dralogues, où la réécriture aura non seulement un effet sur l'authenticité du texte mais aussi sur sa valeur littéraire. Enfin, il faudrait souligner, dans ce remarquable travail d'édition, le soin mis à donner au lecteur l'information la plus complète possible en lui fournissant glossaire, index, bibliographie et des appendices qui éclairent soit la toponymie, soit les noms des divers personnages historiques, soit ceux des tribus indiennes qui, selon les textes et les traditions, sont reconnues sous des noms parfois passablement différents et susceptibles d'ajouter à la confusion d'un lecteur moderne peu rompu à ces variations. Le travail accompli est immense. Compte tenu du format, cela demeure une œuvre qu'il n'est pas facile de se payer et on ne peut que souhaiter que l'œuvre entière de Lahontan soit de nouveau disponible dans une édition bon marché qui renverrait à̀ l'édition critique consultable en bibliothèque.

Au terme de ces réflexions positives, je voudrais relever un certain nombre de questions que pose cette édition. Et je commencerai par les Dialogues qui demeurent la pièce la plus connue de cette œuvre. Si la question de l'originalité est bien mise en lumière, il me semble que l'élaboration de cette œuvre est examinée avec un peu moins d'attention. Je prendrai comme exemple la note 156 de la page 866 . Nous sommes dans le dialogue consacré à la médecine. Adario vient d'affirmer: «Mon Père en a 52: et j'en ay 35 [ans]». La note explique: "En 1688, au moment où Lahontan situe ses dialogues, Kondiaronk avait environ 39 ans. . Tout en étant assez prudente pour ne faire que "situer. les Dialogues, cette note infère tout de même que la date où 
ils sont *situés * pourrait bien être celle où ils ont été écrits. Pourtant, il aurait été facile de faire un autre calcul, plus respectueux du caractère fictionnel de ce texte. L'âge d'Adario pourrait bien être celui de Lahontan: si on ajoute 35 ans à la date de naissance de Lahontan, cela donne 1701, une date assez vraisemblable de l'écriture des Dialogues. Ce ne serait pas la première fois que ce procédé est utilisé. Juste auparavant d'ailleurs, Adario n'avait-il pas aussi affirmé: «Mon GrandPère que tu prends pour un homme de soixante-dix ans en a 98." Or, le père de Lahontan a soixante-dix ans à la naissance de ce dernier... Nous voulons simplement souligner que les Dialogues pourraient être une création récente au moment de leur publication, une création qui aurait alors pu être influencée par des lectures postérieures au voyage. D'ailleurs, dans l'Introduction, Réal Ouellet parle de la lecture possible des Dialogues de Lucien dont on vient de publier une nouvelle traduction française en Hollande (p. 33; $\mathrm{n}^{\circ}$ 49). Lorsqu'on cherche à reconstituer l'élaboration de l'œuvre, des détails comme la question des âges peuvent ainsi avoir toute l'utilité du lapsus.

Autre point de curiosité: le caractère fictionnel des Dialogues ne semble pas évident même à l'époque de leur publication. Il est intéressant de se rapporter à la lettre écrite par Leibniz à Bierling, le 10 novembre 1710 , et citée dans l'appendice 1 par les éditeurs: «Les Dialogues de Lahontan, quoiqu'ils ne soient pas totalement vrais, ne sont pourtant pas non plus totalement inventés." On remarquera la construction de la phrase qui privilégie le fictionnel sur le référentiel, mais on est encore beaucoup plus étonné lorsque, plus loin, Leibniz, qui affirme bien connaître Lahontan, écrit à propos d'Adario: ‘ qui vint en France voilà quelques années et qui, même s'il appartient à la nation huronne, jugea ses institutions supérieures aux nôtres.» (p. 1149-1150) Voilà une affirmation bien curieuse d'un homme aussi bien informé que semble l'être Leibniz. Il semble bien que Lahontan entretient lui-même l'équivoque quant à la réalité du voyage en France d'Adario. On sait que Kondiaronk n'est jamais venu en France. Il ne saurait donc être question de lui. Faut-il voir alors dans Adario un personnage composite où on trouverait à la fois la Grangula et quelqu'autre personnage rencontré par Lahontan aux hasards de ses voyages ou ne faut-il voir là qu'une tentative de sa part de donner de la crédibilité à son affabulation? Autre élément où la fiction est très nette, c'est le dialogue sur le mariage. C'est le dialogue où la division entre les deux personnages est moins nette. Lahontan et Adario disent la même chose ou à peu près, car le seul point de vue à être débattu est celui du mariage blanc qu'Adario ne peut connaître. On trouve dans ce dialogue un déséquilibre entre l'habituelle dialectique des 
Dialogues. Il me semble qu'il y aurait eu un certain avantage à montrer comment ils établissent un écart de fictionnalité en fonction des autres écrits. Aussi est-il facile de s'étonner devant la thèse développée par Georges Sioui et que citent les éditeurs reconnaissant Làhontan comme le découvreur de l'^américité ${ }^{3}$. Si la thèse èst en soi intéressante, on voit mal comment elle peut être tirée des seuls Dialogues. Il aurait fallu chercher des preuves équivalentes dans les Nouveaux Voyages et dans les Mémoires. Telle qu'elle est, cette thèse risque de se voir teintée par l'écart fictionnel que nous retrouvons dans les Dialogues et par la volonté de lire en Adario le seul et unique Kondiaronk.

Il y a une autre mise en garde que nous pourrions faire à l'égard de cette édition. Elle concerne, cette fois, le contexte historique. Les éditeurs ont bien raison de se méfier du sort fait à Lahontan tant par les historiens que par les religieux. Ils ont voulu bien démarquer Lahontan de ses principaux contradicteurs. Ce faisant, ils ont peut-être laissé le pendule aller un peu trop dans l'autre sens. L'antagonisme entre Lahontan et les jésuites leur semble tel qu'ils évitent de parler des jésuites et, surtout, de montrer comment Lahontan n'hésite pas à recourir aux mêmes idées et aux mêmes points de vue qu'eux sur les Indiens. Ainsi, par exemple, dans la mise en contexte de la période coloniale, il n'est pas question du rôle des jésuites, de leurs écrits, de leurs difficultés avec le pouvoir civil, et en particulier avec Frontenac. Au moment où Lahontan est en Nouvelle-France, on trouve, outre les jésuites, les récollets, les sulpiciens... On affirme que Lahontan pouvait avoir accès à diverses sources d'information sur place. On peut penser qu'il a bien dû parler à un jésuite ou deux puisqu'on l'imagine difficilement en train de lire la totalité des Relations. Il est au courant de la doxa du milieu et il serait invraisemblable qu'au cours de ses périgrinations, il n'ait pas eu des contacts même amicaux avec certains jésuites. Autre élément qui modifie un peu le contexte historique: les éditeurs citent volontiers des textes contemporains de Lahontan ou encore lui donnent raison ou tort en citant des textes actuels, Eccles, Fenton, Trigger... qui lui donnent tantôt raison, tantôt tort. Or, ces citations mettent parfois en doutent la compréhension des textes. Ces historiens de la "nouvelle" génération ont normalement lu. Lahontan, les jésuites et toutes les archives disponibles. Il est difficile de départager sur tel ou tel point s'ils ne sont pas déjà au départ d'accord avec Lahontan. Les citer revient alors à une pétition de principe. Les citations établies de façon diachronique, mais sans que ne soient justifiés ni les choix ni les idéologies, risquent de donner une vision assez confuse de l'historiographie actuelle. Cela peut être responsable de 
quelques notations erratiques comme le nombre de soldats qui composaient le régiment de Carignan, 1200 hommes à la page 20 et seulement 1000 à la page 265 . Il en va de même pour les différentes références modernes à la situation iroquoise. On retrouve dans une même note Jennings et Hunt qui, pourtant, ne partagent pas le même point de vue. L'effet de la citation, ici, où ils se complètent, gomment les différences plus larges qui les séparent. La méthodologie de la citation n'est pas clairement établie et un lecteur averti aura l'impression d'une unanimité, là où les points de vue diffèrent. Le choix des œuvres citées n'est pas toujours sans parti pris.

Dernier irritant: il me semble qu'il manque quelque chose d'important à cette édition. Il est dit, montré, répété que Lahontan voulait devenir un espion d'abord pour la France, puis pour l'Espagne, puis pour l'Angleterre. Il est affirmé, par Lahontan lui-même, que les Anglais auraient un intérêt à s'emparer de la Nouvelle-France. Tout cela demande des explications qui sont absentes de l'édition. Qu'estce qu'un espion au début du XVIII ${ }^{e}$ siècle? Qu'est-ce qu'on attend de lui? Peut-on imaginer que les pays auxquels Lahontan offre ses services ne sont pas déjà au courant de tout ce qui se trouve dans ces œuvres? Le territoire de la Nouvelle-France est immense, il est ouvert, on y circule comme on veut, les Indiens ont leurs intérêts propres à sauvegarder, alors comment peut-on imaginer que Lahontan puisse ici vraiment servir un pays à titre d'espion? Une réponse serait de remarquer que, sauf peut-être l'Espagne, personne n'a requis ses services. Mais cela est trop facile. Ou bien l'espionnage n'est rien de plus et Lahontan apparaît comme un farfelu, ou bien il s'agit d'un autre type d'espionnage plus engagé sur les structures de l'État. L'édition est muette sur cette fonction qui pourtant traverse la seconde partie de l'existence de Lahontan, soit une vingtaine d'années... Et on se prend à rêver quand on lit, dans la chronologie, que Jean Talon était un des témoins au mariage de son père.

En conclusion, l'édition critique des œuvres de Lahontan a l'immense mérite de nous restituer un personnage fascinant, une œuvre parfois brillante, mais aussi très intriguante qui n'a pas encore livré son dernier mot. Le lecteur contemporain ne peut sortir de cette lecture sans une multitude de questions sur un personnage qui a connu ses quelques heures de gloire tant en Nouvelle-France qu'en Europe, qui a compté parmi ses amis des gens comme Leibniz et quelques têtes couronnées, tout en demeurant à la poursuite d'une richesse qui lui a toujours échappé, et dont le reste de la famille s'est recyclé dans les maisons louches et peut-être le crime pendant que lui rêvait à l'espionnage. Il nous a laissé une rivière Longue dont nous ne 
sommes pas tout à fait certains et un personnage hors de mesure, Adario, sage Huron, ancêtre des Hurons d'aujourd'hui, ou peut-être plutôt un personnage composite qui se drape en sauvage pour mieux cacher les pensées audacieuses de ce jeune aventurier, qui mourra sans avoir fait ses pâques, mais en ayant quand même manifesté l'intention. Justement, Lahontan est un homme d'intentions, un petit aristocrate écorché dont le titre s'amenuise avec l'âge. Il n'en est pas moins un témoin important de l'établissement de la Nouvelle-France et un personnage littéraire que cette édition ramène heureusement au sein de l'institution.

1. Lahontan, CEuvres complètes, I et II, édition critique par Réal Ouellet et Alain Beaulieu, Montréal, Presses de l'Université de Montréal, .Bibliothèque du Nouveau Monde :, 1990.

2. Il faudrait ici noter un phénomène curieux de l'édition. En restituant Lahontan et en replaçant Gueudeville au niveau des variantes - et je n'ai pu vérifier si ce travail avait été exhaustif - , on perd un peu le sens de l'influence de Lahontan, positive ou négative, que la réécriture de Gueudeville avait engendrée. En somme, certains éléments de la critique adressée à Lahontan seraient peut-être disparus s'il n'y avait pas eu la *variante Gueudeville.

3. Voir en particulier le cinquième chapitre de Pour une autobistoire amérindienne, Québec, Presses de l'Université Laval, 1989. 